\title{
Design and Operation of an Open Interoperable Automated Demand Response Infrastructure for Commercial Buildings
}

\author{
Mary Ann Piette, Girish Ghatikar, Sila Kiliccote, David Watson \\ Lawrence Berkeley National Laboratory \\ Ed Koch, Dan Hennage \\ Akuacom
}




\section{DISCLAIMER}

This document was prepared as an account of work sponsored by the United States Government. While this document is believed to contain correct information, neither the United States Government nor any agency thereof, nor The Regents of the University of California, nor any of their employees, makes any warranty, express or implied, or assumes any legal responsibility for the accuracy, completeness, or usefulness of any information, apparatus, product, or process disclosed, or represents that its use would not infringe privately owned rights. Reference herein to any specific commercial product, process, or service by its trade name, trademark, manufacturer, or otherwise, does not necessarily constitute or imply its endorsement, recommendation, or favoring by the United States Government or any agency thereof, or The Regents of the University of California. The views and opinions of authors expressed herein do not necessarily state or reflect those of the United States Government or any agency thereof or The Regents of the University of California. 


\title{
Design and Operation of an Open, Interoperable Automated Demand Response Infrastructure for Commercial Buildings
}

\author{
Mary Ann Piette, Girish Ghatikar, Sila Kiliccote, David Watson \\ Lawrence Berkeley National Laboratory \\ Ed Koch, Dan Hennage \\ Akuacom
}

\begin{abstract}
This paper describes the concept for and lessons from the development and field-testing of an open, interoperable communications infrastructure to support automated demand response (autoDR). Automating DR allows greater levels of participation, improved reliability, and repeatability of the DR in participating facilities. This paper also presents the technical and architectural issues associated with auto-DR and description of the demand response automation server (DRAS), the client/server architecture-based middle-ware used to automate the interactions between the utilities or any DR serving entity and their customers for DR programs. Use case diagrams are presented to show the role of the DRAS between utility/ISO and the clients at the facilities.
\end{abstract}

Keywords: demand response, automation, commercial, industrial buildings, peak demand

\section{Introduction}

Demand response (DR) is a set of activities that reduce or shift electricity used to improve electric grid reliability, manage electricity costs, and provide systems that encourage load shifting or shedding during times when the electric grid is near its capacity or electric prices are high. Demand response has been identified as an important element of the State of California's Energy Action Plan II, which was developed by the California Energy Commission (CEC), California Public Utilities Commission (CPUC), et al. [1,2]. DR has been identified as a key national strategy to improve electricity markets and electric grid reliability [3]. DR is also being planned as an important feature to improve grid reliability as more intermittent supply such as solar energy and wind power are added to the grid.

Recent experience with DR has shown that customers have limited knowledge of how to operate their facilities to reduce their electricity costs under critical peak pricing (CPP) or other DR programs [4]. While the lack of knowledge about how to develop and implement DR control strategies is a barrier to participation in DR programs like CPP, another barrier is the lack of automation of building systems. Most DR activities are initialed with manual notification and require building operations staff to first receive electronic mails, phone calls, and pager signals, and second, to be physically present to act on notification to execute DR strategies.

The various levels of DR automation can be defined as follows. Manual demand response involves a labor-intensive approach such as manually turning off or changing comfort set points at each equipment switch or controller. Semi-automated demand re- sponse involves a preprogramed DR strategy initiated by a person via centralized control system. Fully automated demand response does not involve human intervention, but is initiated at a home, building, or facility through receipt of an external communications signal. The receipt of the external signal initiates preprogramed DR strategies. The authors refer to this as auto-DR or open auto-DR to emphasize the open, nonproprietary data model. One important concept in auto-DR is that a homeowner or facility manager should be able to "opt out" or "override" a DR event if the event comes at time when the reduction in end-use services is not acceptable. 
From the customer side, modifications to the site's electric load shape can be achieved by modifying end-use loads. Examples of DR strategies include reducing electric loads by dimming or turning off noncritical lights, changing comfort thermostat set points, or turning off noncritical equipment. These DR activities are triggered by specific notifications set by the electricity service provider, depending on parameters such as dynamic pricing or demand bidding. Many electricity customers have suggested that automation will help them institutionalize their DR. The alternative is manual DR - where building staff receives signal and manually reduce demand. Lawrence Berkeley National Laboratory's (LBNL) research has found that many building energy management and controls systems (EMCSs) and related lighting and other controls can be preprogramed to initiate and manage electric DR.

This paper provides an overview of the auto-DR field tests and implementation activities from 2003 to 2007 along with an overview of the technology design. This paper draws heavily on results reported in previous papers [5-8]. This paper also presents the initial Use cases associated with efforts underway to make the DR automation server (DRAS) interface a standard that is interoperable for use for DR with commercial buildings.

\section{Related Research}

During the course of the development and field-testing of auto-DR we tracked related activities around the United States and abroad. Spees and Lave [9] discussed the lack of technology as a barrier in adoption of DR and that automation technology for customer response may lower costs. While many DR aggregators have proprietary automation technology, there is no common, open, interoperable system like auto-DR. A related activity is IntelliGrid, which is an effort led by the Electric Power Research Institute (EPRI) that started in 2004. The IntelliGrid initiative seeks to integrate available basic technologies to create a resilient and responsive intelligent power grid. The IntelliGrid system is based on an open architecture and is a comprehensive technical framework that links communications infrastructure and electricity markets into a "smart grid" that can create a flexible power system for utilities to integrate electric data with a computing infrastructure. The IntelliGrid program is working to advance common industry standards, protocols, and technologies in collaboration with EPRI's "Living Laboratory," a demonstration facility that allows utilities to test communication and data interoperability for metering and control systems to consider both energy efficiency and DR [10].

Another related activity is by a nonprofit consortium, the Organization for the Advancement of Structured Information Standards (OASIS). OASIS is working on "the development, convergence, and adoption of open standards for the global information society" and Open Building Information Exchange (oBIX) standards [11]. Originated in April 2003, the oBIX initiative was started by Continental Automated Buildings Association (CABA), and is now managed under the OASIS consortium. OASIS/oBIX technical committee emphasizes integration of mechanical and control systems in buildings with enterprise applications and functions. This process includes defining web services protocol standards to achieve control systems integration.

The Gridwise Architecture Council (GWAC) is a consortium of private and public stakeholders working toward developing architecture of "highly intelligent and interactive electric systemone ripe with decision-making information exchange and market-based opportunities" (12). This architecture is used to allow interoperability among different technologies and interaction among participants. The GWAC itself is not a design or standards body and only identifies potential areas that allow standardization and interoperability.

Two other efforts are important to mention. The International Electro-Technical Commission (IEC) has developed an international standard for designing substation automation. IEC 61850 
describes data models that can be integrated with varying protocols for communication networks and systems in substations [13]. The Distributed Management Task Force has defined standards for a common information model (CIM). The CIM definitions manage and exchange "information within systems, networks, applications, and services" for vendor interoperability. These standards are defined in the form of an open specification and schema [14]

Our review of previous work has found that the unique aspect of the DR automation we have developed is that it enables end-to-end open interoperable communications between a utility or an independent systems operator (ISO) and end-use customers. Another unique feature of our technology is that it is designed to provide both electricity price information plus emergency or reliability signals.

\section{Technology History}

The auto-DR project began in 2002 following California's electricity market crisis with the goal of addressing three key research questions. First, is it possible using today's technology to develop a low-cost, fully automated infrastructure to improve DR capability in California? Second, how "ready" are commercial buildings to receive common signals? Third, once a building receives a signal, what type of strategies is available that can be readily automated? Research planning began in 2002 and a series of field tests and implementation programs was organized to advance the technology from the initial conceptual design to the status today where it has been designed for use with over 100 commercial buildings over $200 \mathrm{~kW}$.

\section{3: Initial Development and Tests}

The 2003 technology development began with the design of a fictitious price signal and automation server using data transmission that could be represented in extensible markup language (XML) as information exchange to support interoperable signaling. The automation uses a scalable client/server architecture and has been tested with both pull and push communication models. Five facilities were recruited: (1) a large office, (2) supermarket, (3) pharmaceutical research campus including a cafeteria and a small office, (4) data center/office, and (5) a university campus library. In recruiting facilities for the initial auto-DR tests we wanted to evaluate different types of facilities, multiple vendor energy information systems [15], multiple vendor EMCS, multiple technology gateways, different types of ownership, and a variety of enduse demand reduction strategies [5].

All of these initial sites had participated in DR and had been equipped with new communications and monitoring systems as part of California's enhanced automation program [16]. Preparations for the test involved the development of an automation server and the XML software client installations at each of the client sites. The client listens to the signal continuously to request DR

event and price level information. The test resulted in fully automated shedding during two events with an average peak reduction of about $10 \%$.

\section{4: Scaled-Up Tests With Relay}

The design of the 2004 tests began with the consideration that many facilities did not have energy information systems (EISs) or EMCS that support XML [15]. We reviewed existing technologies and protocols such as Modbus and modified the DR automation server, called price-server system (PSS), to enable automation of EMCS using a low-cost Internet relay. The Internet relay is a device with relay contacts that can be actuated remotely over a local or wide area network or the Internet using Internet protocols (IPs). The 2004 technology development and field tests were similar to the 2003 tests in that they were purely fictitious, with no real payment for DR performance. Eighteen sites were recruited to participate in a series of tests. To help in recruiting, the facility managers were offered the assessment of how ready their automation systems were to 
receive common signals for the future's dynamic tariffs and DR program opportunities.

Figure 1 shows the geographic distribution of the participant sites along with the development sites and price-server and clients. Many development sites for the XML software client were located outside of California. The price clients listening to the signals could be implemented outside California. Energy managers out of state can monitor the automation system communications in real time from any web browser using the Internet.

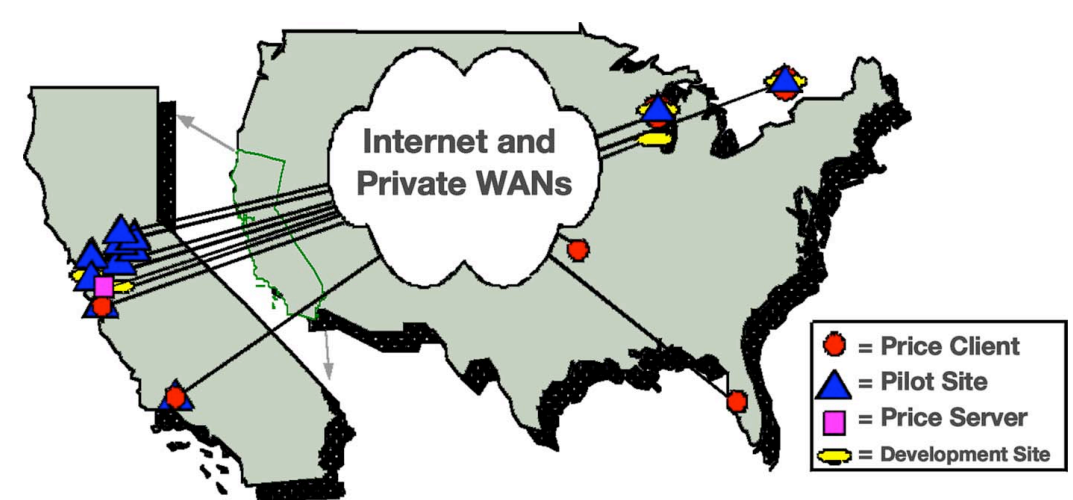

Fig.1 Geographic location of auto-DR facilities, automation clients, and server

With this distributed service oriented architecture (SOA), the price-server, clients, and facilities can be located in diverse geographic locations. Fifteen facilities participated in the 2004 tests with about half using the XML software client and half using the Internet relay. The average demand reduction for these 15 sites was $0.05 \mathrm{~W} / \mathrm{m} 2$ or about $14 \%$ of the whole building electric-peak demand. Table 1 shows an example of how a building would preprogram a response to general DR mode information. A facility manager can decide how to translate the general DR modes into whatever response strategy they choose.

Though version 1.0 served the needs of the initial research program, it also highlighted the need for improvements in the system. During the summer DR test periods, the PSS 1.0 was only available about $90 \%$ of the time. In several cases, the system failed intermittently during a DR event. One participating site was particularly hard-hit by the PSS failures. During a $6 \mathrm{~h}$ DR event, the PSS failed by inappropriately switching in rapid succession between normal and shed modes. This caused the air dampers of approximately 10,000 variable air volume boxes to rapidly change between minimum and wide open. While this was occurring, the facility energy manager received text notifications on his pager that the system was "entering shed mode" then "entering normal mode" and so on. These messages were sent while the manager was helplessly engaged in an important meeting. Another shortcoming of the PSS 1.0 was the level of effort and difficulty required in order for programmers to write interface software to site-specific client software. This software was required for all sites that used software gateways between the PSS and the facility EMCS to reduce heating ventilating and air-conditioning (HVAC) or lighting equipment loads. With the PSS 1.0, the time required to develop interface client software varied from few days to a few weeks.

\section{5: Critical Peak Pricing}

In 2005 we began our initial collaboration with the Pacific Gas and Electric Co. (PG\&E) to offer auto-DR as part of the CPP program. To participate, a site had to be willing to go onto PG\&E's CPP tariff. The tariff offers a rate discount during most summer days, but prices increase on CPP days as shown in Fig. 2. Time of use (TOU) electricity rates vary with the time of day, day of 
week, and season and may include demand charges $(\$ / \mathrm{kW})$. Fifteen facilities participated in the automated CPP tests. CPP usually is called 12 times each summer but because the automation systems took time to install, the tests were not conducted until late in the summer. For the eight sites that participated in the fully automated CPP event on September 29, 2005, the average DR ranged from $0 \%$ to $24 \%$ per site for the medium price period and $4 \%$ to $28 \%$ per site during the high-price period, with an average of $9 \%$ and $14 \%$ overall for the two price periods.

The 2005 automated CPP tests used a new automation server renamed as the DRAS. This server was operated at a secure industrial grade hosting facility and was designed to accommodate future availability and scalability criteria. DRAS version 2.0 was built from scratch to meet the high standards and security using Internet technology. The DRAS successfully met the requirements for the 2005 tests including the following.

1. Flexibility. The system was customized to interface with PG\&E's existing CPP processes and ITRON's InterAct-II ${ }^{\mathrm{TM}}$ system.

2. High availability/reliability. The system successfully processed every PG\&E initiated autoCPP event. Since coming online in 2005 through the end of the 2006 DR season, DRAS version 2.0 has never had an unexpected downtime event, thus exceeding the availability target of $99.99 \%$. The DRAS is hosted at a secure colocation facility with triple redundant back-up, uninterrupted power supply (UPS), and generator systems.

3. Scalability. Tests show that the 2.0 framework was more than adequate for the 20 sites supported in the 2005 and 2006 pilots. Preliminary scalability tests indicate that the current system could support approximately 30,000 "sites" per server with an end-to-end latency averaging less than 1/2 s. Modular software is designed to enable support of millions of sites by simply adding servers, load balancers, and other standard information technology (IT) equipment.

4. Security. The DRAS architecture was designed so as to be secure enough to meet current industry standards for financially binding transactions. Secure socket layer (SSL) technology with 128 bit encryption and authentication certificates are used to assure the same high levels of security used by banks and other financial institutions worldwide.

Table 1 Sample DR building control strategies by mode

\begin{tabular}{lcccc}
\hline \hline Building type & End use & Normal & Shed Level-1 moderate & Shed Level-2 high \\
\hline $\begin{array}{l}\text { Large office } \\
\text { Supermarket }\end{array}$ & HVAC & $\begin{array}{c}\text { Zones-22 C } \\
\text { All on }\end{array}$ & $\begin{array}{c}\text { Zones-24 C } \\
\text { Lights down 35\% }\end{array}$ & Antisweat night mode \\
\hline \hline
\end{tabular}




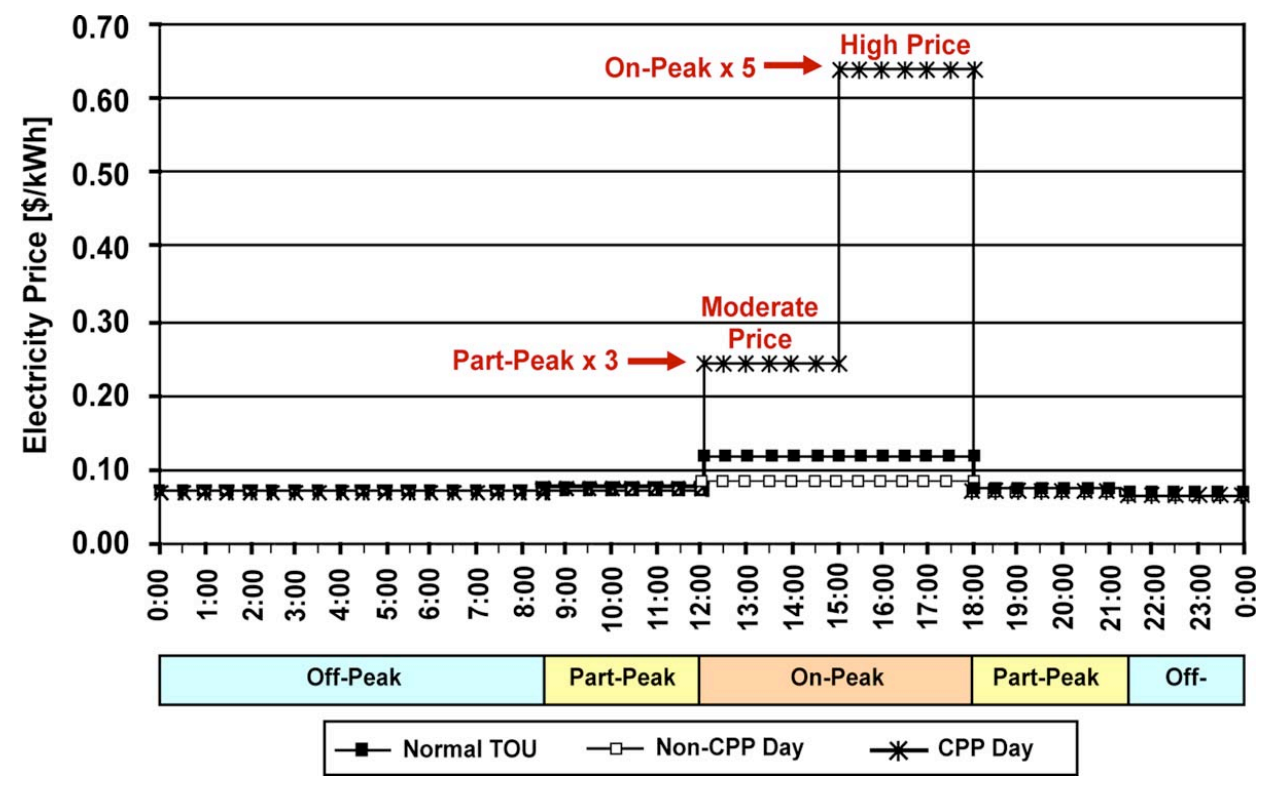

Fig. 2 Critical peak price tariff compared with TOU

\section{6: Scaled-Up Automated CPP}

Following the pilot automated CPP test in 2005 we began a formal partnership with PG\&E's emerging technologies program [7]. In an effort to transfer the expertise of the automation system installation efforts from the LBNL to a third party, we developed a qualification procedure for third-party engineering services. Initially named the DR Integration Services Co., or DRISCO, this service company was renamed in 2007 to an auto-DR technical coordinator. In addition to recruiting new sites into the program, we had about 11 sites that had fully automated CPP response for the entire summer with 12 events. More importantly, we provided this automation system through a severe heat wave in July 2006. Each site continued to reduce their demand over many days during this 1.5 week event. None of the sites opted out or overrode the automation capability, although that option was available. Figure 3 below shows an auto-DR shed at an office building in Martinez California. The shed shows a classic response with the first level of response based on resetting the zone temperatures up a few degrees, and second level reset response during the $3 \mathrm{~h}$ high-price period. Over $100 \mathrm{~kW}$ was shed during the high-price period with no rebound peak demand when the building goes into unoccupied mode after 6 p.m. Among the auto-CPP sites, site responses to 125 events were fully automated and evaluated in this study. The average peak demand reduction was $14 \%$ of the whole-facility load based on the $3 \mathrm{~h}$ high-price period [8]. 


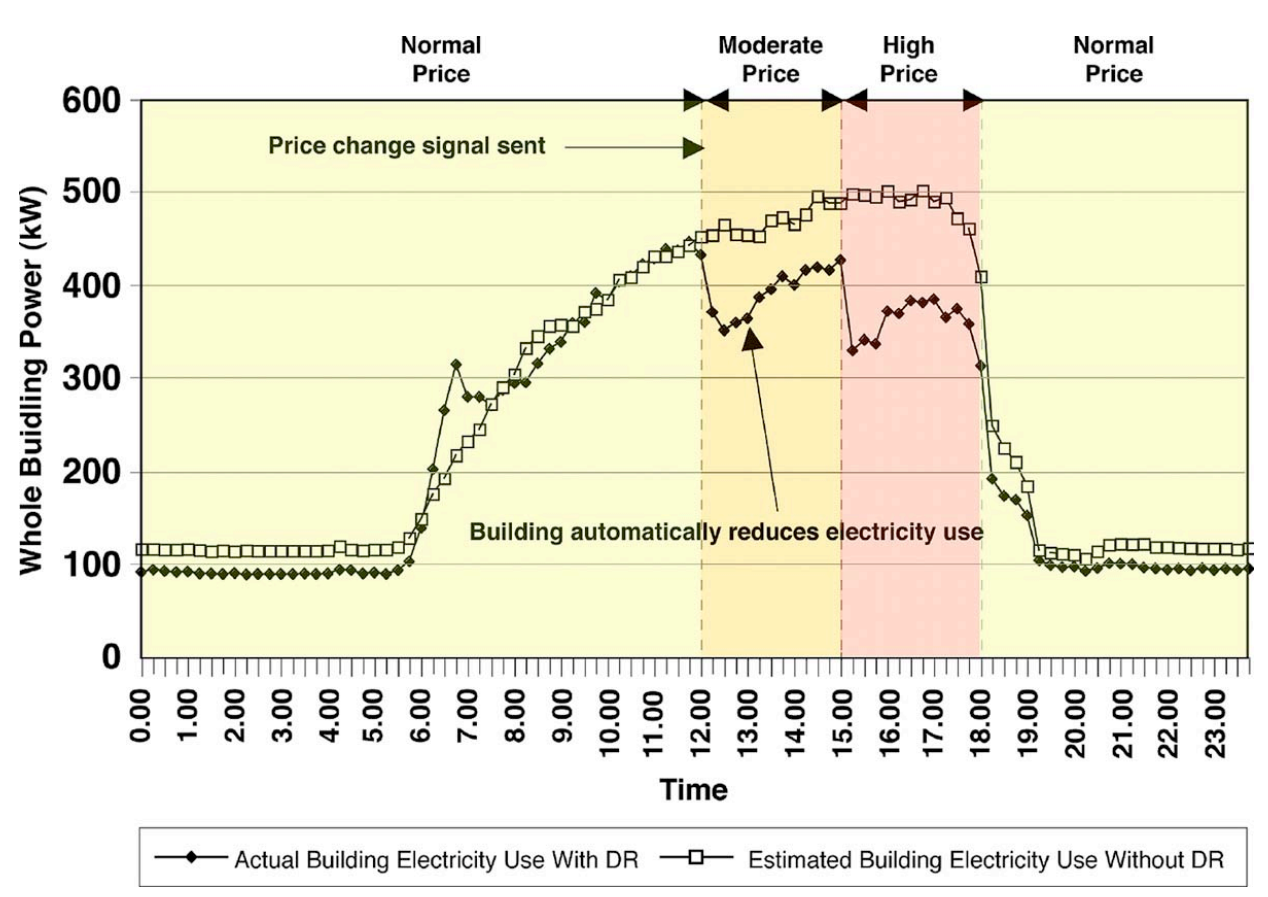

Fig, 3 Examples of load shape change with auto-DR at Martinez, CA Office Building Electricity Use with and without auto-DR: June 21, 2006 (outdoor air maximum temperature: $39^{\circ} \mathrm{C}$ )

The electricity cost savings are typically a few percent of the annual energy costs, but are highly variable depending on the shape of the whole-facility loads compared with the CPP price schedule.

As we brought the technology out to a large customer base we found that the Internet relay had communications security issues for some customers. A hole in the corporate network firewall was often needed to allow the server to push the signal into the relay. As a result of that finding, a new DRAS client was developed. This technology, known as the client and logic with integrated relay or CLIR, was developed as an IT friendly "plug and play" automation client as further discussed below. It is typically installed inside of the secure enterprise network and "polls" for CPP event information using 128-bit SSL encryption and authentication using Hypertext Transfer Protocol (HTTPS), commonly known as HTTP secure or HTTPS. SSL, as in HTTPS, is also used for most online financial transactions. In most cases, no modification to corporate enterprise firewall or local area network (LAN) is required when using the CLIR. The web services (WSs) DRAS client application programming interface (API) was also available to facilities to program and embed the client within the advanced control systems that can communicate XML and IP.

\section{DR Automation System Features}

Following the hot summer of 2006 the CPUC requested the three California investor-owned utilities (IOUs) to begin using auto-DR technologies [8]. As part of that effort we developed a more formal definition of auto-DR to outline the principles for the automation system design. Auto-DR for commercial and industrial facilities can be defined as a fully automated DR initiated by a signal from a utility or other appropriate entity and provide fully automated connectivity to customer end-use control strategies.

- - Signaling. Auto-DR technology should provide continuous, secure, reliable, two-way communication with customers to allow end-use sites to be identified as listening and acknowledging receipt of DR signals. 
- Industry standards. Auto-DR consists of open, interoperable industry-standard control andcommunications technologies designed to integrate with both common energy management and control systems and other end-use devices that can receive a dry contact relay or similar signals (such as Internet based XML).

- Timing of notification. "Day ahead" and "day of" signals are provided by auto-DR technologies to facilitate a diverse set of end-use strategies such as precooling for day ahead notification, or near real-time communications for day of control strategies. Auto-DR communications must consider weekends and holidays for day ahead events. The auto-DR architecture has five steps as shown in Fig. 4 below.

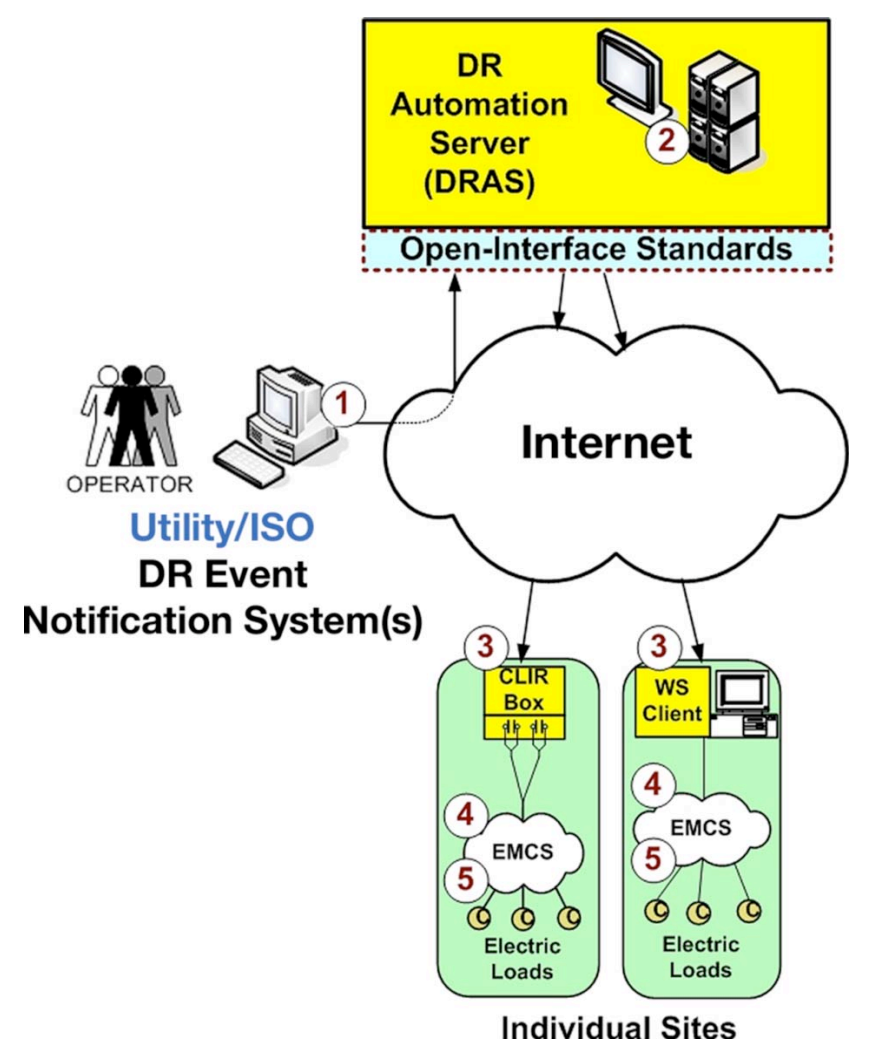

Fig. 4 Automated demand response general features

The steps are as follows.

1. The utility/ISO defines DR event and price signals sent to the DRAS.

2. DR event and price services published on the DRAS.

3. DRAS clients (CLIR or WS) request latest event or price information from the DRAS every minute.

4. Customized preprogramed DR strategies determine action based on DR event price or mode.

5. Facility EMCS or related controls carry out shed based on DR event signals and strategies.

The PG\&E auto-DR program was expanded in 2007 to include both CPP and demand bidding. The demand bidding program (DBP) allows more customer participation because it is voluntary and the sites do not need to go onto the PG\&E CPP tariff. Even the direct-access sites can participate in DBP. The bidding automation uses a standing DR bid that triggers an automated response whenever the program is called. The facilities also have the option to change their standing bids once the event is issued and before the request for bids closes and override the event participation. The 2007 PG\&E auto-DR program also included recruitment and technical 
coordinators to market, evaluate, configure, and manage the automation systems. Over $22 \mathrm{MW}$ of demand reduction have been recruited into the program.

The DRAS is designed to generate, manage, and track DR signals between utilities/ISOs to aggregators and end-use customers and their control systems that perform various shed strategies in response to the DR signals. Each facility or end-use customer hosts a DRAS client that is responsible for bridging communications between the DRAS and the automated system (e.g., EMCS) responsible for controlling electricity consumption. It may be a "smart" software-based client implemented with an existing subsystem or a "simple" dedicated piece of hardware whose responsibility is to proxy communications between the DRAS and the EMCS. The CLIR box in Fig. 4 depicts the latter. The DRAS clients are discussed in greater detail in Sec. 4.1.

\section{DRAS Clients}

The DRAS clients interface between the DRAS on the utility/ISO side and the EMCS or other systems on the customer side. The DRAS client typically exists on the customer side and can vary for implementations within an aggregator model.

\section{Web Services' Software Client}

Many newer or advanced EMCS or lighting control systems within facilities can communicate using WS and transmission control protocol (TCP)/IP and are interoperable with native protocols. In such cases, a WS API is available to facilitate programmers to write software programs to communicate to the DRAS and respond to events using preprogramed DR strategies. These WS software clients can be embedded directly within the control systems, thus eliminating the need for any gateway or CLIR. In some cases the WS DRAS software client has been installed using gateway systems. An Internet gateway typically connects TCI/IP to the native protocol of a given EMCS.

\section{Modbus Internet Relay Client}

Rather than require sites to have a standard direct digital control (DDC) based Internet gateway, another connectivity option in form of Modbus native protocol Internet relay was provided for the 2004 and 2005 tests. If desired, LBNL provided participating sites with this low-cost Internet relay. The Internet relay is based on a standard protocol (TCP/IP) and all EMCS can sense the state of relay contact closures (regardless of their particular EMCS protocol). Because of this, Internet relays can be used on virtually any commercial building that has a standard connection to the Internet. Internet connectivity directly to the EMCS is not required and was used to remotely signal many of the sites for the auto-CPP tests. Instead of converting XML messages to the native EMCS protocol, the Internet relay simply closes relay contacts, which were read as digital inputs by EMCS controllers. The in-house staff programed the EMCS to curtail loads based on the state of the Internet relay mappings. The simplicity and ease of the Internet relay architecture made it possible for many sites to participate in the 2005 auto-DR tests that would not have been able to do so otherwise. However, there were some impediments to the use of the Internet relay.

- Requires coordination with the IT group at the site. In few very secure LAN environments, the IT group must make some minor configuration changes to the corporate firewall and network configurations to enable the DR automation server to push messages to the Internet relay.

- The firewall and network reconfiguration is a task that may take $1-3 \mathrm{~h}$ plus substantial time for coordination and authorization.

- Though practically without merit, the firewall and network reconfiguration can be perceived as a risk to the security of the network, and many corporate network policies do not allow it. 


\section{CLIR}

The CLIR is a secure, self-configuring Internet relay client and connects an EMCS over the site LAN to the DRAS over the Internet. The CLIR hosts a WS software client and converts the event signals received from DRAS using TCP/IP to the legacy EMCS through a standard relay contacts. At most times, no reconfiguration of the site firewall is required. Predetermined DR strategies are programed into the EMCS based on the relay mappings. The EMCS then responds to price or contingency-based events generated in the DRAS and communicated via the CLIR. The CLIR was proven in the field to overcome impediments of prior DRAS clients. Although the hardware cost is currently about $\$ 1500$, reduced configuration labor and increased security features in majority of the networking environments make CLIR the best connectivity option for most commercial participants. Additional CLIR development and improved installation procedures and wide-scale use are expected to further lower the production and the installed cost of connectivity. In 2007, only CLIR and WS DRAS clients were supported.

The implementation and operation of the DRAS clients have been fairly trouble free. Installation at a few sites required troubleshooting due to the incorrect configuration of network parameters such as IP and connectivity issues. Such problems were fixed using reconfigured parameters that provided an improved connection. Overall the system has performed reliably with minor communications failures. Auto-DR problems that have occurred are typically related to problems within the building control systems that are somehow not configured properly to automate the sheds or load shifts. Programming changes, new facility managers, or software upgrades can impact the status of a DR control strategy. In the future, periodic, short duration, pre-DR season tests may be needed to ensure end-to-end functionality of automation in preparation for summer or winter DR events.

\section{Use Cases}

The DRAS is designed to support two major classes of utility/ISO and participant interactions: DR event notification and automated bid submission. The use of DRAS in each of these functions is detailed in this section.

\section{Automated DR Event Notification.}

Almost all DR programs require participants to respond to DR events from the utility/ISO, which are normally handled by human operators. The main concept of auto-DR is to remove the humans from the loop as much as possible and thus automating the actions within the facilities. The DRAS accomplishes this by brokering the communications between the utility/ISO and the equipment in the facilities. This is depicted in the automated DR event notification as shown in the use case diagram of Fig. 5 below. 


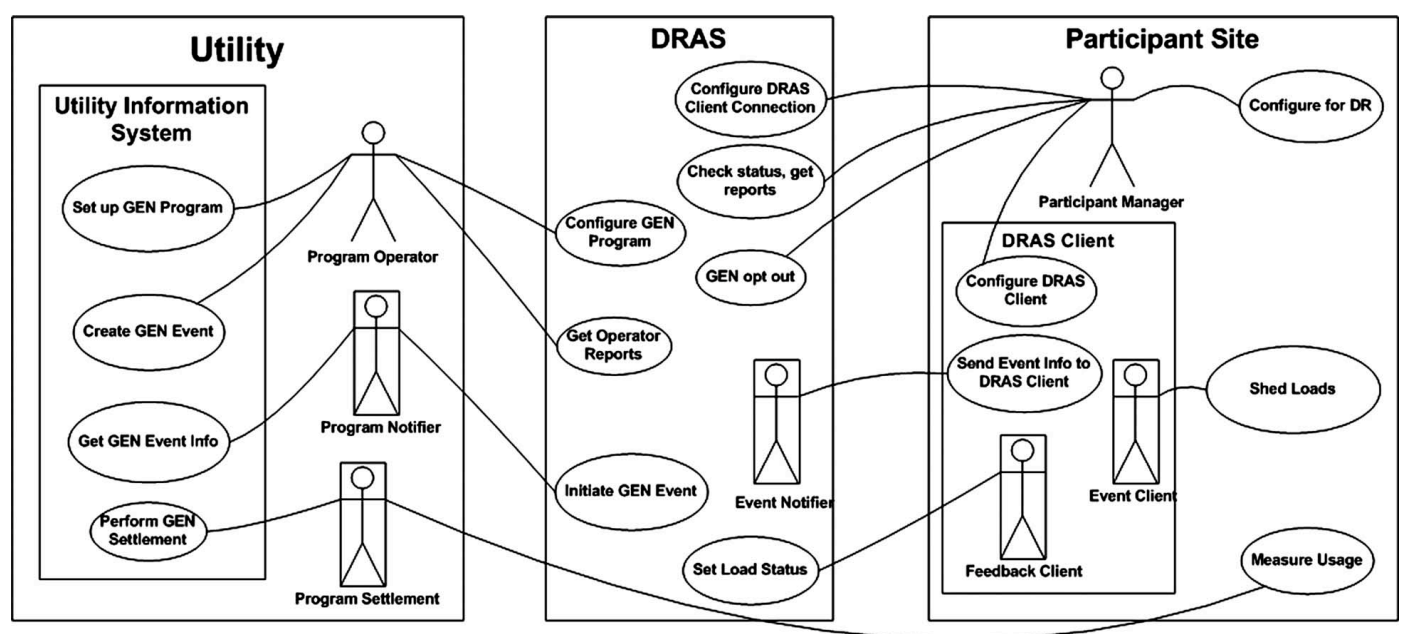

Fig. 5 Event notification use case

The sequence of operations that take place when the utility/ISO issues a DR event is the following.

1. Utility program operator creates generic (GEN) DR event in utility information system.

2. Utility program notifier gets GEN DR Event information from utility information system (date and time) and initiates GEN DR event in DRAS.

3. Event notifier in DRAS sends event info to all DRAS clients in DR program.

4. DRAS event client in facility sends event info to client subsystems resulting in the shedding of loads.

5. DRAS feedback client in facility sets load status in DRAS (e.g., shed status information).

6. Utility program settlement measures usage in client sites and performs settlement in utility information system.

In addition to specific DR events the DRAS is also designed to handle real-time pricing (RTP) streams from the utility/ISO and potentially convert these into DR events for the facility to act upon.

Note that a number of ancillary operations are also performed in support of DR event notifications including configuration, operations, and reports. The DRAS also supports these activities although they are not described in detail in this paper.

\section{Automated Bid Submissions}

Some DR programs require that participants submit bids for available shed resources. The utility/ISO will then either accept or reject those bids and those that are accepted will receive subsequent DR event notifications to perform the actual sheds. The submission of bids is yet another DR related activity that requires a human in the loop and is thus a candidate for further automation.

Experience has shown that many participants in these types of DR programs rarely change their bids from one DR event to another. Thus using the concept of a "standing bid," DRAS can be used to automate the submission of bids. The participants can program standing bids into the DRAS and whenever the utility/ISO issues a request for bids the standing bids can be submitted automatically by the DRAS at the appropriate time. Figure 6 below shows the use case diagram to automate standing bid submission by customers. 


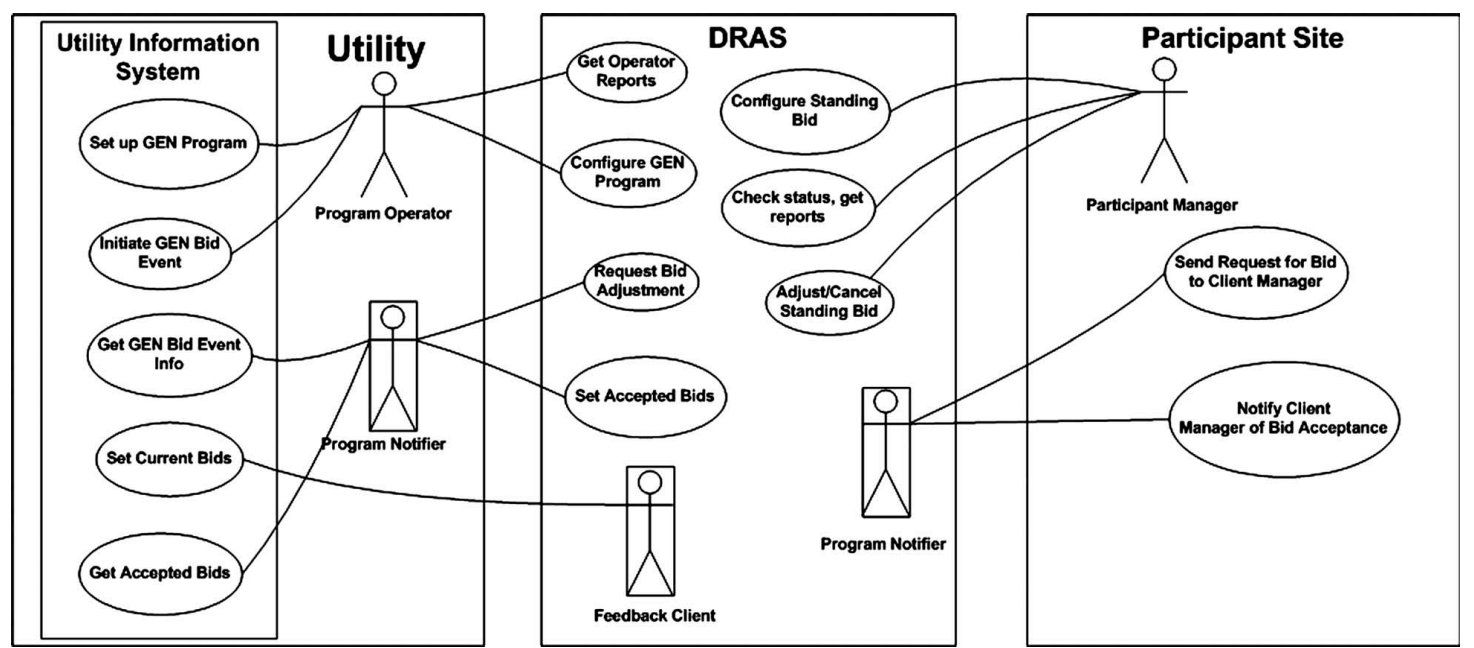

Fig. 6 Automated bidding use case

The sequence of steps used to perform automated bid submissions is the following.

1. Utility program operator initiates generic (GEN) bid event in utility information system.

2. Utility program notifier gets GEN bid event information from utility information system (date and time) and initiates a request for bid adjustment in DRAS (request for bids).

3. DRAS program notifier sends request for bid to the participant manager.

4. Participant manager adjusts/cancels current bid in DRAS (optional).

5. After specified time limit the DRAS bidding proxy sets the current bid in the utility information system.

6. Utility program notifier gets accepted bids from utility information system and sets accepted bids in DRAS.

7. DRAS Program Notifier sends the acceptance notification to the Client Manager

\section{DRAS Open Interface Standardization}

The use of common standards for the various DRAS interfaces would have the benefit of interoperability, ease of implementation, and lower the effort and cost of implementing auto-DR programs and tariffs, and thus increases the level and reliability of participation among them. In 2007 the DRRC began standardization efforts by bringing together a consortium of industry stakeholders primarily composed of the major California utilities and ISO. In addition other industry experts and research and standards organizations such as CEC, EPRI, Building Automation Control Network (BACnet) subcommittee, National Institute for Standards and Testing (NIST), California Institute of Energy and Environment (CIEE), University of California Berkeley (UCB), Open Automated Metering Infrastructure (OpenAMI), and Open Home Automation Network (OpenHAN) are participating in the effort.

The standardization effort relies heavily on the lessons learned since 2002 in implementing autoDR programs in California. The objective is to have an initial draft of the standard in spring 2008 that can form the basis of a DRAS implementation that can be used in the DR programs in the summer of 2008. It is anticipated that the standard produced by this industry consortium may eventually be submitted to a standards organization such as Institute of Electrical and Electronics Engineers (IEEE) or American Society of Heating, Refrigeration, and Air conditioning Engineers (ASHRAE) to become an official standard and compliance testing. 


\section{DRAS Requirements}

The following are some of the general requirements of the DRAS.

1. Communications with the DRAS should use readily available and existing networks such as the Internet.

2. The DRAS interfaces should be platform independent and leverage existing standards such as XML and web services.

3. The DRAS should use a security policy that enables privacy of the communications using measures such as authentication and encryption.

4. The DRAS should support communications with a variety of control systems that may range from a very simple EMCS to those with sophisticated data processing and programming capabilities.

5. The DRAS should not be dependent on specific control systems within the facilities.

6. DRAS clients that communicate with the DRAS should easily integrate with existing facility networks and IT infrastructures.

7. The DRAS should support aggregated loads that may be managed by third-party aggregators.

8. Reconciliation (or settlement) of DR event participation is outside the scope of the DRAS. There are a number of methods such as aggregators, AMI, etc., that can and will handle the measurement of sheds for the purposes of the reconciliation of DR programs.

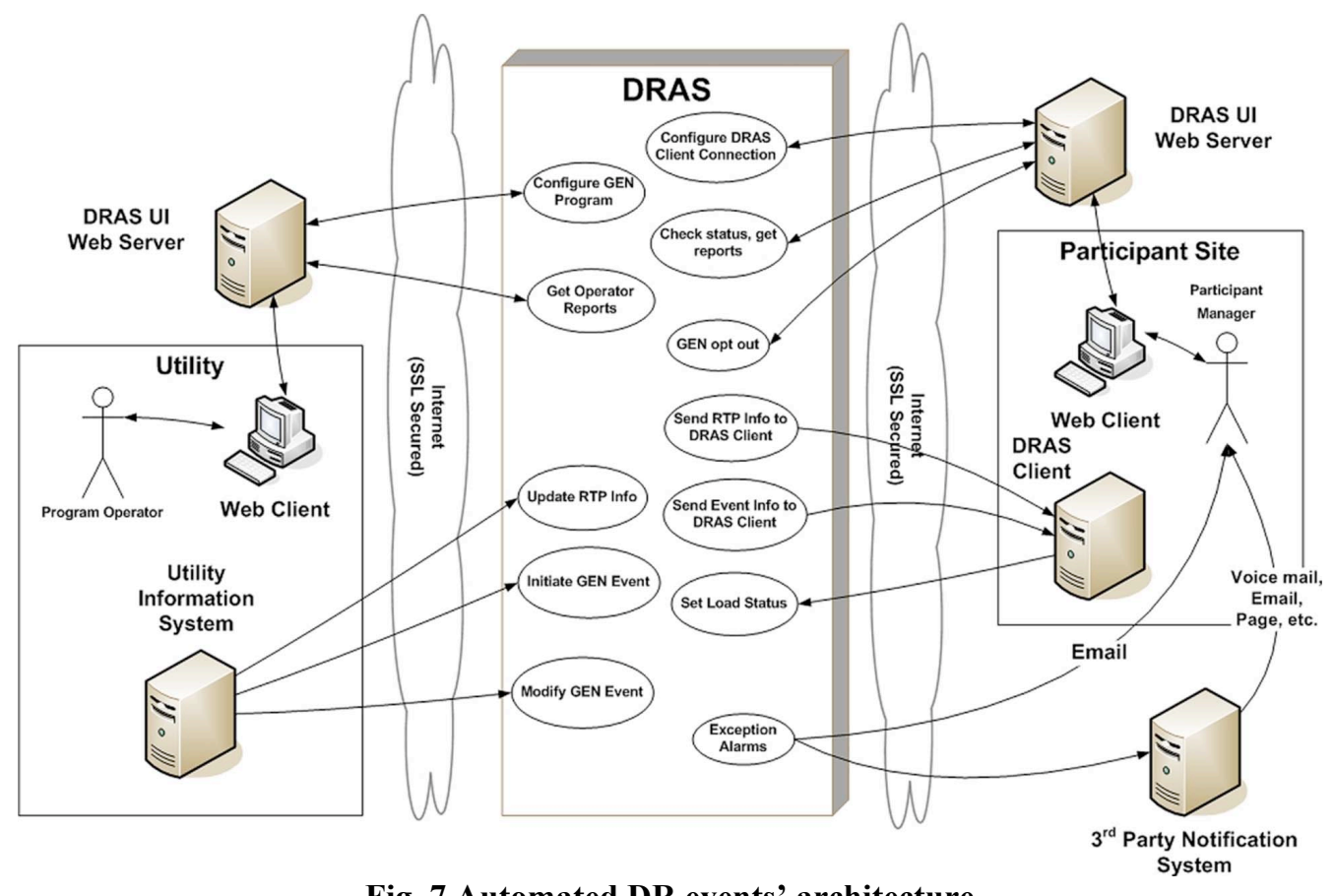

Fig. 7 Automated DR events' architecture

\section{Automated Demand Response System Architecture}

The general architecture for handling automated DR events is shown in Fig. 7. This system is built on industry-standard SOA using secure WS. The DRAS is intended to interface to two different types of DRAS clients within the participant's facility. The first is the classification of WS software as "smart DRAS client," which is capable of receiving full DR event information as specified by the utility/ISO. The second is the classification of CLIR or similar hardware interface as "simple DRAS client," which receives a simplified characterization of the DR event in terms of simple levels such as normal, moderate, and high. The simple DRAS client should be used in environments where there is not a sophisticated EMCS that can be easily programed. 
As shown in Fig. 7 RTP is depicted as being supported in the DRAS. It is anticipated that in the case of smart DRAS clients the RTP information is sent directly to the DRAS clients. In simple DRAS clients there will be a set of rules configured in the DRAS to convert RTP information to the simple levels that the simple DRAS clients require. In Fig. 7 the DRAS is depicted as a standalone component, but it should be understood that the DRAS might be integrated with the utility/ISO or the participants' information systems.

Furthermore the interface with the DRAS client is intended to support both a push and pull model of interaction. The pull model benefits from being easier to integrate with existing IT infrastructures because of firewall issues and security certificates. The push model has the benefit of reduced latency and network activity.

\section{Summary and Future Directions}

This paper has presented the history and status of an open, interoperable auto-DR system. The research began with advanced control and energy information systems that could host XMLbased signals. Recent work has included automating relay signals with Internet based communications in secure, open web services architecture. Research on commercial buildings control strategies has also shown good potential for wide spread DR. Since the auto-DR scope and DRAS design are relatively narrow, they are easily integrated with existing infrastructures and operations for both the utility/ISOs and the participants. With the development of standard interfaces to the DRAS it is hoped that the architecture will become even more widespread and there will be the development of additional DRAS clients that will enable a wider range of facilities to leverage the benefits of DR. Future efforts include standardization of the communications and signaling systems, and efforts to move the technology into future building codes and standards.

\section{Acknowledgment}

The work described in this report was coordinated by the Demand Response Research Center and funded by the California Energy Commission (CEC), Public Interest Energy Research (PIER) Program, under Work for Others Contract No. 500-03-026 and by the U.S. Department of Energy under Contract No. DE-AC02-05CH11231. Special thanks to our research sponsors including Kristy Chew, Mike Gravely, and Martha Brook (CEC). The authors are grateful for the extensive support from numerous individuals who assisted in this project. Special thanks also to Ron Hofmann and Roger Levy for technical support, and to the PG\&E for multiyear support.

\section{References}

[1 [California Energy Commission (CEC), California Public Utilities Commission (CPUC), et al., 2005, "State of California, Energy Action Plan II: Implementation Roadmap for Energy Policies," pp. 3-5, CEC, Jan. 2, http:// www.energy.ca.gov/energy_action_plan/2005-09-

21_EAP2_FINAL.PDF.

[2] California Energy Commission (CEC), 2003, “2003 Integrated Energy Policy Report,” CEC, Jan. 2, http://www.energy.ca.gov/reports/100-03-019F.PDF.

[3] United States General Accounting Office (GAO), 2004, "Electricity Markets: Consumers Could Benefit for Demand Response Programs, But Challenges Remain," GAO, Jan. 2, http://www.gao.gov/new.items/d04844.pdf.

[4] Quantum Consulting Inc. and Summit Blue Consulting, LLC Working Group 2, 2005, "Demand Response Program Evaluation-Final Report," Prepared for Working Group 2 Measurement and Evaluation Committee and Southern California Edison Company, Apr. 28, California Energy Commission, Jan. 2.

[5] Piette, M. A., Sezgen, O., Watson, D. S., Motegi, N., and Shockman, C., 2005, "Development and Evaluation of Fully Automated Demand Response in Large Facilities," Report Nos. CEC- 
500-2005-013 and LBNL-55085.

[6] Piette, M. A., Watson, D. S., Motegi, N., Bourassa, N., and Shockman, C., 2005, "Findings From the 2004 Fully Automated Demand Response Tests in Large Facilities," Report Nos. CEC500-03-026 and LBNL-58178.

[7] Piette, M. A., Watson, D., Motegi, N., Kiliccote, S., and Xu, P., 2006, “Automated Critical Peak Pricing Field Tests: Program Description and Results," Report to the Pacific Gas and Electric Company Emerging Technologies Program and California Institute for Energy and the Environment, Report No. LBNL-59351.

[8] Piette, M. A., Watson, D., Motegi, N., and Kiliccote, S., 2007, "Automated Critical Peak Pricing Field Tests: 2006 Pilot Program Description and Results," Report No. LBNL-62218. [9] Spees, K., and Lave, L. B., 2007, "Demand Response and Electricity Market Efficiency," Electr. J., 20(3), pp. 69-85.

[10] Electric Power Research Institute (EPRI) IntelliGrid Consortium, "The IntelliGrid Consortium: Leading the Drive to Transform the Power Delivery System,” EPRI, Jan. 2, http://www.epri.com/IntelliGrid/.

[11] Organization for the Advancement of Structured Information Standards (OASIS) Consortium, “About OASIS," OASIS, Jan. 2, http://www.oasis-open.org/ who/.

[12] Gridwise Architecture Council (GWAC), 2008, Jan. 2, http://www.gridwiseac.org/.

[13] Communication Networks and Systems in Substations, 2008, International Electro-Technical Commission (IEC) 61850 Standard, Pt. 1-10, May 25, http://www.iec.ch/ and http://tissues.iec61850.com/parts.mspx.

[14] Common Information Model (CIM) Standards, Distributed Management Task Force, Inc. [DTMF], 2008, May 25, http://www.dmtf.org/standards/cim/.

[15] Motegi, N., Piette, M. A., Kinney, S., and Herter, K., 2003, "Web-Based Energy Information Systems for Energy Management and Demand Response in Commercial Building," Report No. LBNL-52510.

[16] California Energy Commission (CEC), 2002, “Technical Options Guidebook: Enhanced Automation," CEC, Jan. 2, http://www.energy.ca.gov/enhancedautomation/documents/400-02005F_TECH_OPTIONS.PDF. 